\title{
Determination of Orders of Relative Alkali Metal Ion Affinities of Crown Ethers and Acyclic Analogs by the Kinetic Method
}

\author{
Chien-Chung Liou and Jennifer S. Brodbelt \\ Department of Chemistry, University of Texas, Austin, Texas, USA
}

\begin{abstract}
Ladders of relative alkali ion affinities of crown ethers and acyclic analogs were constructed by using the kinetic method. The adducts consisting of two different ethers bound by an alkali metal ion, $\left(\mathrm{M}_{1}+\mathrm{Cat}+\mathrm{M}_{2}\right)^{+}$, were formed by using fast atom bombardment ionization to desorb the crown ethers and alkali metal ions, then collisionally activated to induce dissociation to $\left(\mathrm{M}_{1}+\mathrm{Cat}\right)^{+}$and $\left(\mathrm{M}_{2}+\mathrm{Cat}\right)^{+}$ions. Based on the relative abundances of the cationized ethers formed, orders of relative alkali ion affinities were assigned. The crown ethers showed higher affinities for specific sizes of metal ions, and this was attributed in part to the optimal spatial fit concept. Size selectivities were more pronounced for the smaller alkali metal ions such as $\mathrm{Li}^{+}, \mathrm{Na}^{+}$, and $\mathrm{K}^{+}$than the larger ions such as $\mathrm{Cs}^{+}$and $\mathrm{Rb}^{+}$. In general, the cyclic ethers exhibited greater alkali metal ion affinities than the corresponding acyclic analogs, although these effects were less dramatic as the size of the alkali metal ion increased. (J Am Soc Mass Spectrom 1992, 3, 543-548)
\end{abstract}

$\mathrm{T}$ The rich history and growth in host-guest chemistry originates largely from the furst synthesis of crown ethers [1], compounds that serve as remarkable but simple models of more complex biologically relevant hosts [2]. The principles underlying host-guest chemistry form the foundation for molecular recognition [2-6], a phenomenon important in selective biological transport, drug actions, and enzyme functions. Examination of various macrocycles and their complexes with an array of guests has led to a fundamental understanding of host-guest selectivity and binding interactions. Some of the most important developments have stemmed from studies of complexation of alkali metal ions with macrocycles [7-31]. The series of alkali metal ions represents simple spherical guests (see Table 1) for which thermodynamic and kinetic parameters of cation selectivity can be measured systematically. The binding interactions are electrostatic in nature and involve the various heteroatomic donor atoms in the macrocycle.

Several important terms and concepts are used to describe complexation processes. The one most often recognized in host-guest binding is the "optimal spatial fit concept" $[8,10]$, used to describe the importance of cavity size (see Table 1) on the ability of a macrocycle to effectively bind various guests. Selectivity is also based on the spatial arrangement of binding

Address reprint requests to Jennifer $S$. Brodbelt, Department of Chemistry, University of Texas, Austin, TX 78712. sites [22-25]. In fact, macrocyclic hosts can undergo some degree of skeletal deformation $[24,25]$ or form sandwiches $(2: 1$ complexes) $[8,11]$ to accommodate different sizes of guests. In relation to binding specificities, "peak" selectivity is characterized by hosts that have an enhanced capability to discriminate against various guest sizes, whereas "plateau" selectivity is observed for hosts that bind an array of guest sizes with nearly equal affinities [13]. The overall stabilities of the resulting host-guest complexes are influenced by the host topology and the nature and number of binding interactions.

Attention has also focused on the importance of the cyclic nature of the host molecule in increasing the stability constants of complexes [29-31, 34]. The observed enhancement of binding strengths of macrocyclic hosts relative to open-chain analogs is termed the "macrocyclic effect". This effect has both enthalpic and entropic origins. Surprisingly, some acyclic analogs to crown ethers may exhibit similar binding affinities [8] because of a template effect that promotes the acyclic hosts to adopt helical conformations. Acyclic hosts typically exhibit plateau selectivities, and this is attributed to two factors. First, open-chain ethers have large flexibilities that allow high conformational adaptability during metal ion complexation and thus reduced size selectivities are observed [31]. Second, acyclic ethers possessing terminal hydroxyl groups (which are stronger donor sites than those located along the polyether chain [31]) have relative 
Table 1. Radii of alkali ions and cavity sizes of crown ethers

\begin{tabular}{cccc}
\hline Alkali ion & Radius $(\AA))^{\mathrm{a}}$ & Crown ether & Radius $(\AA)^{\mathrm{b}}$ \\
\hline \hline $\mathrm{Li}^{+}$ & 0.76 & $12-\mathrm{C}-4$ & $0.6-0.75$ \\
$\mathrm{Na}^{+}$ & 1.02 & $15-\mathrm{C}-5$ & $0.86-0.92$ \\
$\mathrm{~K}^{+}$ & 1.38 & $18-\mathrm{C}-6$ & $1.34-1.55$ \\
$\mathrm{Rb}^{+}$ & 1.52 & $21-\mathrm{C}-7$ & $1.7-2.1$ \\
$\mathrm{Cs}^{+}$ & 1.67 & & \\
\hline
\end{tabular}

aef 32

Bef 33

alkali metal binding affinities that are predominantly influenced by interactions of the end groups and not by the length of the ether chain [34]. By contrast, acyclic ethers with terminal ether groups (glymes) show more selective complexation but have overall lower binding affinities to alkali metal ions $[31,34]$.

The selectivities and binding strengths of alkali metal ion complexation by various macrocycles and open-chain analogs have been measured in solution by such techniques as calorimetry [14], potentiometry $[19,20]$, conductometry [17], carbon-13 nuclear magnetic resonance [18], fast atom bombardment combined with mass spectrometry (FAB/MS) [35-38], and electrohydrodynamic mass spectrometry (EHMS) [39. 40]. The thermodynamic and kinetic measurements are largely influenced by solvent effects, however, and this makes derivation of intrinsic host-guest selectivities difficult. For example, metal-macrocyclic complexes were found to be more stable in methanol than in water because of the increased solvation competition of water for the alkali metal ions [20]. Moreover, it was reported that macrocycles showed greater selectivities for various sizes of alkali metal ions in dimethylsulfoxide and water than in less polar solvents (tetrahydrofuran, acetone) [18] because of the increased solvation effects. In the less polar solvents, the cavity sizes of the macrocycles played a greater role in the determination of selectivities due to the decreased enthalpies of the alkali metal ion-solvent interactions. In fact, it was suggested that because of the dominance of solvent effects, cavity size could not always be a reliable predictor of selectivity [19]. Many computational studies have recently confirmed that solvent effects can play as important a role as the conformational flexibility of the crown ethers in the selectivities and stabilities of crown-metal complexes [26-28].

Such solvation effects, of course, would be eliminated in the gas phase, and then the intrinsic selectivities of macrocycle-metal ion complexation could be examined. Because of our interest in understanding host-guest chemistry from a solvent-free perspective [41-43], we have undertaken an investigation of metal ion-crown ether interactions in a mass spectrometer. Our initial studies of host-guest chemistry in the gas phase indicated that both chemical and topological effects played important roles in the binding of biologically important molecules such as $\mathrm{O}_{2}$ and $\mathrm{CO}$ to perfluorinated macrocycles. We report herein relative scales of alkali metal ion affinities of crown ethers and acyclic ether analogs determined by using the kinetic method [44, 45]. Mass-spectrometric techniques have been used previously to measure stability constants and selectivities of crown ethers for alkali metal ions $[35-40,46,47]$. For the most part, these studies used FAB/MS or EHMS to directly desorb or electrically extract metal-crown ether complexes formed in a glycerol solution [35-38], and thus the thermodynamic parameters measured for the complexation processes were largely influenced by solvation effects. One recent study [47] has shown that crown-metal ion sandwiches can be formed via gas-phase ionmolecule reactions, and size-dependent chemistry was observed. We have recently completed a study of the gas-phase selectivities of crown ethers for alkali metal ion complexation [48]. The present study involves the evaluation of alkali metal ion binding affinities of crown ethers as determined from sandwich complexes isolated in the gas phase.

\section{Experimental}

A Finnigan triple stage quadrupole mass spectrometer (TSQ-70); Finnigan-MAT, San Jose, CA) was used to evaluate the gas-phase alkali metal ion affinities of the crown ethers. The crown ethers were mixed with a selected alkali metal salt, and this mixture was applied to the tip of a FAB probe. Ions and neutrals were desorbed from the probe by xenon fast atom bombardment gun. The FAB source was an Ion Tech gun with a $B 50$ power supply operated at $8 \mathrm{kV}$. The manifold pressure was typically $1.0-9.0 \times 10^{-6}$ torr. The abundances of $\left(\mathrm{M}_{1}+\mathrm{Cat}+\mathrm{M}_{2}\right)^{+}$ions relative to $(\mathrm{M}+\mathrm{Cat})^{+}$ions are $1-5 \%$. The kinetic method was used to determine the relative ligand affinities of the crown ethers $[44,45]$. Using this method, a correlation is made between metal affinities and favored dissociation routes of the adducts, $\left(\mathrm{M}_{1}+\mathrm{Cat}+\mathrm{M}_{2}\right)^{+}$. For instance, a metal-bound adduct ion is selected with the first quadrupole, then passed through the second quadrupole filled with $<0.6$ mtorr of argon. The collision energy, defined by the potential difference between the ion source and the second quadrupole, was set between 2 and $5 \mathrm{eV}$. The collision energy and pressure were kept low to approach threshold dissociation conditions. Upon collisional activation, the adducts dissociate only to $\left(\mathrm{M}_{1}+\mathrm{Cat}\right)^{+}$, $\left(\mathrm{M}_{2}+\mathrm{Cat}\right)^{+}$, and occasionally $\mathrm{Cat}^{+}$ions of very low abundance, with up to $30 \%$ total conversion effciency. At higher collision energies and pressures, the observed ratios of fragments remained substantially unchanged. The triple-quadrupole mass spectrometer was operated in the constant precursor transmission mode. In all cases, the mass-to-charge ratios of the fragment ions were sufficiently similar to neglect significant mass discrimination effects. The ratio of $\left(\mathrm{M}_{n}\right.$ $+\mathrm{Cat})^{+}$ions formed is directly related to the relative affinities of each crown ether for the specified alkali metal ion. Typically, there are no other competitive 
dissaciation pathways observed after the collisional activation step, and this offers some evidence that only the alkali-metal binding interactions are disrupted, not the skeletal structures of the ethers. Furthermore, the ratios of fragment ions observed from collisional-activation dissociation of a specific adduct are not dependent on the concentration of metal salt or crown ether in the source. The kinetic method has been used for studies of complex multifunctional compounds with multiple binding sites, such as deoxynucleosides [49] and amino acids [50]. For these other reports and for the present one, it is assumed that the frequency factors for cleavages of the multiple binding interactions are similar, and the multiple collision environment for adduct formation (from FAB) approaches thermal equilibrium. Finally, the relative affinities are estimates, only as accurate as can be expected from the kinetic method and its known shortcomings.

All compounds except 21-crown-7 were obtained from Aldrich Chemical Company (Milwaukee, WI) and used without further purification. The 21-crown-7 was obtained from Parish Chemical Company (Orem, UT). The ethers used in this study are shown in Table 2 along with their structures. The number of oxygen donor atoms is indicated because this represents the number of binding sites and hence influences the relative binding strengths of the ethers to the alkali metal ions.

\section{Results and Discussion}

An example of the methodology used to determine relative alkali metal ion affinities is illustrated in Figure 1 . The potassium-bound adduct ion of pentaethylene glycol (5-GLYCOL) and 15-crown-5 is shown in Figure 1a as a single ion peak at $m / z$ 497. This ion was passed through the second quadrupole for collisional activation. The resulting fragment ions (15- crown- $5+\mathrm{K})^{+}$and $(5-\mathrm{GLYCOL}+\mathrm{K})^{+}$are shown in Figure $1 \mathrm{~b}$. The ratio of the abundances of these ions indicates that pentaethylene glycol has a higher $\mathrm{K}^{+}$ affinity than 15-crown-5 (the relative affinity ratio is about 2.5:1). Other pairs of compounds gave other results. In this systematic fashion, ladders of alkali metal ion affinities were constructed (see Table 3).

\section{Orders of Relative Affinities}

The relative affinity scale is different for each metal ion, but the biggest variation occurs for the metal ions with the smallest ionic radii. For instance, there are striking changes in the ladders constructed for $\mathrm{Li}^{+}$. $\mathrm{Na}^{+}$, and $\mathrm{K}^{+}$, but the orders for $\mathrm{Rb}^{+}$and $\mathrm{Cs}^{+}$are nearly identical to the one observed for $\mathrm{K}^{+}$. This leveling in the variations of affinities for the $\mathrm{K}^{+}, \mathrm{Rb}^{+}$, and $\mathrm{Cs}^{+}$ions is attributed to size effects. These cations have sufficiently large diameters and corresponding lower charge densities that the most effective multiple bonding interactions, especially within the cavities of the crown ethers, are less size-dependent [18-20].

The first trend of particular interest is the relative size selectivities of the crown ethers. Examination of the orders shown in Table 3 indicates that the crown ethers exhibit special affinities for certain sizes of metal ions. For example, 15-crown-5 has the highest $\mathrm{Li}^{+}$affinity among all the ethers, but its relative rank drops for $\mathrm{Na}^{+}$, then decreases again for the larger alkali metal ions. By contrast, both 18-crown-6 and 21-crown-7 have lower relative $\mathrm{Li}^{+}$ion affinities, but their relative affinities are larger for $\mathrm{Na}^{+}$, and then their affinities remain at the top of the scale for $\mathrm{K}^{+}$, $\mathrm{Rb}^{+}$, and $\mathrm{Cs}^{+}$. The placement of 12 -crown-4 on the ladders never changes; it always sets the lower limit for alkali metal ion affinities. These trends in relative affinities of the crown ethers can be attributed in part to cavity sizes and the optimal spatial fit concept. There appears to be an optimal metal ion size that

Table 2. Ethers and number of oxygen donor atoms

\begin{tabular}{|c|c|c|c|}
\hline Ether & Abbrevation & Structure & $\begin{array}{l}\text { Number } \\
\text { of donor } \\
\text { atoms }\end{array}$ \\
\hline 12-crown-4 ${ }^{a}$ & $12-\mathrm{C}-4$ & $\square\left[\mathrm{CH}_{2} \mathrm{CH}_{2} \mathrm{O}\right]_{4} \sqsupset$ & 4 \\
\hline 15-crown-5 & $15-\mathrm{C}-5$ & $\square\left[\mathrm{CH}_{2} \mathrm{CH}_{2} \mathrm{O}\right]_{5} \sqsupset$ & 5 \\
\hline 18-Grown-6 & $18-C-6$ & $\square\left[\mathrm{CH}_{2} \mathrm{CH}_{2} \mathrm{O}\right]_{6} \sqsupset$ & 6 \\
\hline 21-crown-7 & $21-\mathrm{C}-7$ & $\square\left[\mathrm{CH}_{3} \mathrm{CH}_{2} \mathrm{O}\right], \square$ & 7 \\
\hline Triethylene glycol dimethyl ether & 3-GLYME & $\mathrm{CH}_{3} \mathrm{O}\left[\mathrm{CH}_{2} \mathrm{CH}_{2} \mathrm{Ol}_{3} \mathrm{CH}_{3}\right.$ & 4 \\
\hline Tetraethylene glycol dimethyl ether & 4-GLYME & $\mathrm{CH}_{3} \mathrm{OlCH}_{2} \mathrm{CH}_{2} \mathrm{Ol}_{4} \mathrm{CH}_{3}$ & 5 \\
\hline Tripropylene glycol dimethyl ether & 3-PROGLYME & $\mathrm{CH}_{3} \mathrm{O}\left[\mathrm{CH}_{2} \mathrm{CH}_{2} \mathrm{CH}_{2} \mathrm{Ol}_{3} \mathrm{CH}_{3}\right.$ & 4 \\
\hline Tetraethylene glycol & 4-GLYCOL & $\mathrm{HO}\left[\mathrm{CH}_{2} \mathrm{CH}_{2} \mathrm{Ol}_{4} \mathrm{H}\right.$ & $5^{\mathrm{b}}$ \\
\hline Pentaethylene glycol & 5-GLYCOL & $\mathrm{HO}\left[\mathrm{CH}_{2} \mathrm{CH}_{2} \mathrm{Ol}_{6} \mathrm{H}\right.$ & $6^{\mathrm{b}}$ \\
\hline
\end{tabular}

\footnotetext{
"The crown ether designation "a-crown- $b$ " indicates the total number of atoms $(a)$ and the number of oxygen atoms $(b)$ in the ring.

${ }^{b}$ The glycols have two terminal hydroxyl groups rather than all ether oxygen donor atoms.
} 


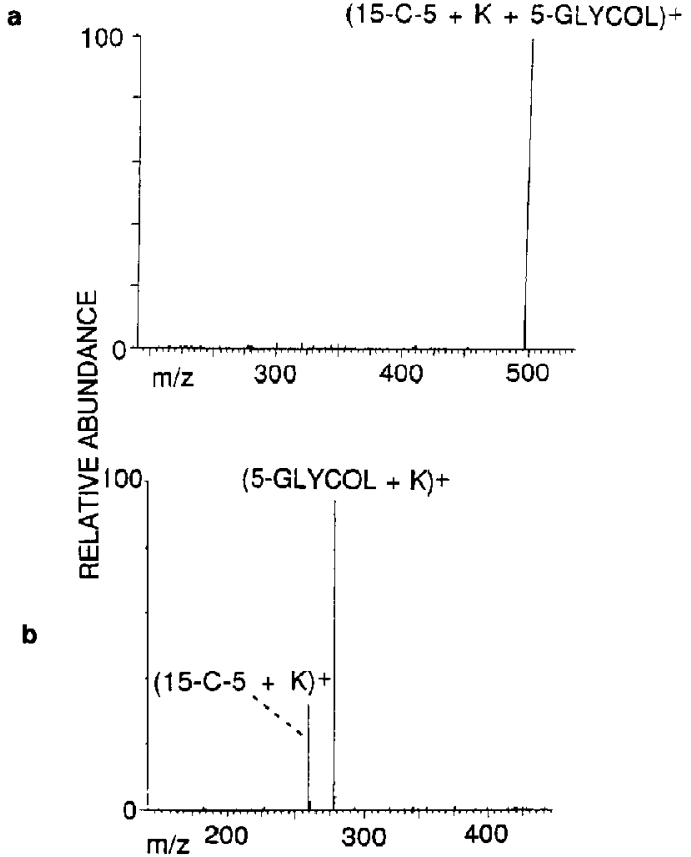

Figure 1. (a) Mass-selected spectrum of the (15-C-5 + K + 5GLYCOL) ${ }^{+}$adduct. (b) Collision-activated dissociation of the $(15-\mathrm{C}-5+\mathrm{K}+5-\mathrm{GLYCOL})^{+}$adduct.

binds most effectively within the cavity of the crown ether or slightly outside the cavity and permits the most favorable multiple bonding interactions $[8,10]$. For the gas-phase measurements, the trends suggest that each crown ether shows greatest affinities for ion sizes that fall within the cavity size range shown in Table 1 , or not more than $0.2 \AA$ greater than the upper limit or $0.2 \AA$ less than the lower limit. The fact that 12-crown-4 does not show a larger relative affinity even for $\mathrm{Li}^{+}$, the smallest alkali metal ion, is surprising but likely is due to the close proximity of the oxygen donor sites, which prevent formation of near-linear electrostatic bridges to the metal ion within the cavity [17].

The fact that 15 -crown-5 has the highest $\mathrm{Li}^{+}$ion affinity of all the ethers and also has a higher $\mathrm{Na}^{+}$ affinity than 18-crown-6 clearly underscores the significance of cavity size effects. The structural units of the various crown ethers are sufficiently similar that the differences in affinities cannot be attributed to differences in electronegativities of the donor atoms but are more consistent with topological variations in the complexes. For $\mathrm{K}^{+}, \mathrm{Rb}^{+}$, and $\mathrm{Cs}^{+}, 21$-crown-7 and 18-crown-6 show higher affinities than other ethers. This suggests that these crowns have suffciently large cavity sizes to promote the most favorable multiple bonding interactions to the cations.

The second trend of interest involves the comparison of affinities of cyclic ethers to acyclic ethers in order to determine the importance of the macrocyclic effect in enhancing binding affinities. The acyclic ethers were chosen to have a similar number of donor atoms as the crown ethers, and two with terminal hydroxyl groups were also compared. Two trends are apparent within the group of acyclic ethers. First, the glymes (methoxy end groups) generally have higher affinities than the analogous glycols (hydroxy end groups). This is attributed to the greater polarizability

Table 3. Orders of relative alkali metal ion affinities of ethers ${ }^{a}$

\begin{tabular}{lllll}
\hline \multicolumn{1}{c}{$\mathrm{Li}^{+}$} & \multicolumn{1}{c}{$\mathrm{Na}^{+}$} & \multicolumn{1}{c}{$\mathrm{K}^{+}$} & \multicolumn{1}{c}{$\mathrm{Rb}^{+}$} & $\mathrm{Cs}^{+}$ \\
\hline $12-\mathrm{C}-4$ & $12-\mathrm{C}-4$ & $12-\mathrm{C}-4$ & $12-\mathrm{C}-4$ & $12-\mathrm{C}-4$ \\
$(1)$ & $(1)$ & $(1)$ & $(1)$ & $(1)$ \\
$3-G L Y M E$ & $3-\mathrm{GLYME}$ & $3-\mathrm{PROGLYME}$ & $3-\mathrm{PROGLYME}$ & $3-\mathrm{PROGLYME}$ \\
$(10)$ & $(2)$ & $(2)$ & $(1)$ & $(1)$ \\
$3-\mathrm{PROGLYME}$ & $3-\mathrm{PROGLYME}$ & $3-\mathrm{GLYME}$ & $3-\mathrm{GLYME}$ & $3-\mathrm{GLYME}$ \\
$(20)$ & $(2.2)$ & $(5)$ & $(5)$ & $(10)$ \\
$4-\mathrm{GLYCOL}$ & $4-\mathrm{GLYCOL}$ & $4-\mathrm{GLYCOL}$ & $4-\mathrm{GLYCOL}$ & $4-\mathrm{GLYCOL}$ \\
$(80)$ & $(20)$ & $(10)$ & $(50)$ & $(40)$ \\
$18-\mathrm{C}-6$ & $4-\mathrm{GLYME}$ & $15-\mathrm{C}-5$ & $15-\mathrm{C}-5$ & $15-\mathrm{C}-5$ \\
$(200)$ & $(200)$ & $(40)$ & $(500)$ & $(250)$ \\
$21-\mathrm{C}-7$ & $18-\mathrm{C}-6$ & $5-\mathrm{GLYCOL}$ & $4-\mathrm{GLYME}$ & $4-\mathrm{GLYME}$ \\
$(200)$ & $(600)$ & $(100)$ & $(1000)$ & $(300)$ \\
$5-\mathrm{GLYCOL}$ & $15-\mathrm{C}-5$ & $4-\mathrm{GLYME}$ & $5-\mathrm{GLYCOL}$ & $5-\mathrm{GLYCOL}$ \\
$(600)$ & $(800)$ & $(120)$ & $(2000)$ & $(300)$ \\
$4-\mathrm{GLYME}$ & $5-\mathrm{GLYCOL}$ & $18-\mathrm{C}-6$ & $18-\mathrm{C}-6$ & $18-\mathrm{C}-6$ \\
$(800)$ & $(1100)$ & $(1000)$ & $(3000)$ & $(600)$ \\
$15-\mathrm{C}-5$ & $21-\mathrm{C}-7$ & $21-\mathrm{C}-7$ & $21-\mathrm{C}-7$ & $21-\mathrm{C}-7$ \\
$(1200)$ & $(4200)$ & $(3000)$ & $(6000)$ & $(1800)$ \\
\hline
\end{tabular}

a Order of increasing affinity down the column, alkali metal ion affinities relative to $12-\mathrm{C}-4$ given in parenthesis. Uncertainties in all values are $\pm 25 \%$. 
of the methyl groups over the hydrogens and the corresponding more favorable ion-dipole interactions between the alkali metal ion and the ether oxygen. Second, just as was observed for the crown ethers, the biggest changes in the relative trends occur for the complexes involving the smallest metal ions. For the larger metal ions, the variations in the trends for $\mathrm{K}^{+}$, $\mathrm{Rb}^{+}$, and $\mathrm{Cs}^{+}$are negligible.

A template effect appears to be operative in rationalizing why 4-GLYME has nearly as high a $\mathrm{Li}^{+}$ affinity as 15-crown-5. The acyclic 4-GLYME has lower $\mathrm{Li}^{+}$and $\mathrm{Na}^{+}$affinities than the corresponding cyclic ether (15-crown-5), but for the larger alkali sizes the 4-GLYME has a greater affinity than 15-crown-5. The 15-crown-5 cavity is too small to accommodate the larger alkali metal ions as effectively, but the acyclic ether has substantial flexibility to adopt a conformation in which multiple bonding interactions remain favorable. The 3-GLYME always has a greater metal affinity than the corresponding cyclic ether (12-crown4), and this is also attributed to its better conformational adaptability.

\section{Characterization of Structures}

To evaluate the types of ions formed upon dissociation of the alkali-metal bound adducts, the structures of the simple polyether/metal ion complexes, $(\mathrm{M}+$ Cat) ${ }^{+}$, formed directly in the ion source during the $\mathrm{FAB}$ process were characterized by collision-activated dissociation at a range of collision energies and collision gas pressures. The objective was to provide some evidence that the ethers, whether acyclic or cyclic initially, maintained their skeletal integrity during the complexation and dissociation process. The $(\mathrm{M}+$ Cat) ${ }^{+}$ions dissociate exclusively to the metal ion Cat ${ }^{+}$ after collisional activation under all conditions. No fragments corresponding to cleaved ether units attached to the $\mathrm{Cat}^{+}$are observed, supporting the assumption (but not proving conclusively) that the skeletal structure of the ether remains intact and is eliminated during the activation process. This suggests that the $(M+C a t)^{+}$are loosely bound species in which the metal-ether bonds are weaker than the covalent bonds of the ether. Such a representation of the crown-metal ion complexes is consistent with the nature of host-guest binding interactions in solution [2-5].

The structures of the initial metal-bound adducts have not been unambiguously determined; however, based on the trends in affinities, it is reasonable to conclude that the adducts may exist initially as sandwich-type structures. This would permit multiple binding interactions between the two ethers and metal ion prior to dissociation and would account for the striking crown selectivities. Linear adducts would prohibit such interactions. Presently, we are undertaking calculations of these adduct structures by using several computational methods [26-28].

\section{Comparison with Solution Results}

A vast compilation of stability constants for crown ether-metal ion complexes in solution exists in the literature [15]. Thermodynamic values have been recorded by using a variety of methods and media [22-25, 51-53]. Some general comparisons can be made to the present gas-phase results. Solution results indicate that 12-crown-4 complexes have the lowest stability constants of all the cyclic ethers, and the selectivity of 12-crown-4 is also lowest [51]. In less polar solvents, such as propylene carbonate, the 15crown $-5 / \mathrm{Li}^{+}$complex has the highest stability constant compared to the other metal ion/15-crown-5 complexes, and the stability constant decreases with the size of the cation [52]. For 18-crown-6, the $\mathrm{K}^{+}$and $\mathrm{Rb}^{+}$complexes generally have the largest stability constants, and for 21-crown-7, the $\mathrm{Rb}^{+}$and $\mathrm{Cs}^{+}$complexes are most stable $[52,53]$. The trends observed in the less polar solvents in which alkali metal ion solvation effects are less significant typically parallel those observed in this gas-phase study.

The complexation of crown ethers and open-chain analogs have been compared in solution [20]. For example, it was found that 18-crown-6/metal ion complexes were much more stable than 5-GLYME/metal ion complexes, and this was attributed to a macrocyclic effect [20]. As noted in the discussion, the gas-phase crown ethers typically have larger relative metal ion affinities than the corresponding acyclic analogs, but some discrepancies are observed, depending on the cavity size of the crown of interest and the size of the metal ion.

\section{Conclusions}

The kinetic method has proved useful for obtaining a qualitative understanding of alkali metal ion affinities of crown ethers and open-chain analogs in the gas phase. Ladders of the relative affinities for the various alkali metal ions show that cavity size effects are important in determining the binding strengths of the crown ether complexes. However, acyclic analogs demonstrate affinities for the alkali metal ions that are similar (albeit somewhat smaller) than the crown ether affinities, and this is attributed in part to the conformational adaptability of the flexible open-chain structures. Relative to solution host-guest chemistry, the trends observed in the gas phase most closely parallel those obtained from complexation studies performed in nonpolar solvents.

It is difficult to make quantitative comparisons between solution results and the present gas-phase results because the solution studies typically evaluated stability constants and selectivities by varying the metal ion with a single crown ether, whereas in the present study the relative affinities of a series of crown ethers for a single metal ion were measured. This issue has been resolved in a related study [48] in 
which the kinetic method was used to measure the selectivities (rather than relative affinities) of crown ethers for different metal ions.

\section{Acknowledgments}

Support from the Welch Foundation (F-1155), NIH (R01 GM46723-01), and an ACS-PRF grant (22270-G5) is gratefully acknowledged.

\section{References}

1. Pederson, C. J. J. Am. Chem. Soc. 1967, 89, 7017.

2. Hilgenberger, R.; Saenger, W. In: Host-Guest Complex Chemistry: Macrocycles; Vogtle, F.; Weber, E., Eds.; SpringerVerlag: New York, 1985, ch. 2.

3. Cram, D. J. Science 1988, 240, 760 .

4. Lehn, I. M. Angew. Chem. Int. Ed. Engl. 1988, 27, 89.

5. Sutherland, I. Chem. Soc. Rev. 1986, 15, 63.

6. Vogtle, F.; Weber, E., Eds. Host-Guest Complex Chemistry: Macrocycles; Springer-Verlag: New York, 1985.

7. Takeda, Y. Top. Curr. Chem. 1984, 121, 1.

8. Weber, E.; Vogtle, F. Top. Curr. Chem. 1981, 98, 1.

9. Cram, D. J.; Trueblood, K. N. Top. Curr. Chem. 1981, 98, 43.

10. Izatt, R. M.; Eatough, D. J.; Christensen, J. J. Struct. Bonding 1973, 16, 161.

11. Mallinson, P. R.; Truter, M. R. J. Chem. Soc. Perkin Trans. 2 1972, 1818.

12. Vogtle, F.; Weber, E. In: The Chemistry of the Ether Linkage, Supplement E; S. Patai, Ed.; Wiley: London, 1981, p 59.

13. Lehn, J. M. Struct. Bonding 1973, 16, 1.

14. Izatt, R. M.; Terry, R. E.; Haymore, B. L.; Hansen, L. D.; Dalley, N. K.; Avondet, A. G.; Christensen, J. J. I. Am. Chem. Soc. 1976, 98, 7620 .

15. Izatt, R. M.; Bradshaw, J. S.; Nielson, S. A.; Lamb, J. D.; Christensen, J. J.; Sen, D. Chem. Rev. 1985, 85, 271.

16. Wong, K. H.; Konizer, G.; Smid, J. J. Am. Chem. Soc. 1970, 92,666 .

17. Buschmann, H. J. J. Soln. Chem. 1987, 16, 181.

18. Mosier-Boss, P. A.; Popov, A. I. J. Am. Chem. Soc. 1985, $107,6168$.

19. Michaux, G.; Reisse, J. J. Am. Chem. Soc. 1982, 104, 6895

20. Frensdorff, H. K. J. Am. Chem. Soc. 1971, 93, 600.

21. Arnett, E. M.; Moriarty, T. C. J. Am. Chem. Soc. 1971, 93, 4908.

22. Lehn, J. M. Angew. Chem. 1988, 27, 89.

23. Hori, K.; Yamada, H.; Yamabe, T. Tetrahedron 1983, 19, 67

24. Live, D.; Chan, S. I. J. Am. Chem. Soc. 1976, 98, 3769.

25. Dunitz, D. J.; Seiler, P. Acta. Cryst. B. 1974, 30, 2739.

26. Wipff, G.; Weiner, P.; Kollman, P. J. Am. Chem. Soc. 1982, $104,3249$.
27. Drew, M. G. B.; Yates, P. C. Pure and Appl. Chem. 1989, 61, 835.

28. Mazor, M. H.; McCammon, J. A.; Lybrand, T. P. J. Am. Chem. Soc. 1990, 112, 4411.

29. Cabbiness, D. K.; Margerum, D. W. I. Am. Chem. Soc. 1969, 91, 6540.

30. Hancock, R. D.; McDougall, G. J. J. Am. Chem. Soc. 1980, 102,6551 .

31. Sieger, H.; Vogtle, F. Angew. Chem. Int. Ed. Engl. 1978, 17, 198.

32. Shannon, R. D. Acta Crystallogr., Sect. A: Found. Crystal$\log r .1976,32,751$.

33. Dalley, N. K. In: Synthetic Multidentate Macrocyclic Compounds; Izatt, R. M.; Christensen, J. J., Eds.; Academic Press: Orlando, 1978, $\mathrm{p} 217$.

34. Vogtle, F.; Weber, E. Angera, Chem. Int. Ed. Engl. 1979, 18, 753.

35. Johnstone, R. A. W; Lewis, I. A. S. Int. I. Mass Spectrom. Ion Phys. 1983, 46, 451.

36. Bonas, G.; Bosso, C.; Vignon, M. R. J. Incl. Phen. Mol. Recogn. in Chem. 1989, 7, 637.

37. Johnstone, R. A. W.; Lewis, I. A. S.; Rose, M. E. Tetrahedron 1983, 39, 1597.

38. Henis, N. B. H.; Youngless, T. L.; Bursey, M. M. Inorg. Nucl. Chem. Lett. 1980, 16, 141.

39. Chan, K. W S.; Cook, K. D. Macromolecules 1983, 16, 1736.

40. Man, V. F.; Lin, J. D.; Cook, K. D. I. Am. Chem. Soc. 1985, 107,4635

41. Brodbelt, J. S.; Maleknia, S.; Liou, C. C.; Lagow, R. J. Am. Chem. Soc. 1991, 113, 5913.

42. Brodbelt, J. S.; Maleknia, S.; Lagow, R.; Lin, T. Y. J. Chem. Soc. Chem. Commun. 1991, 23, 1705.

43. Malkenia, S.; Liou, C. C.; Brodbell, J. S. Org. Mass Spectrom. 1991, 26, 997 .

44. Cooks, R. G.; Kruger, T. L. I. Am. Chem. Soc. 1977, 99, 1279.

45. McLuckey, S. A,; Schoen, A. E; Cooks, R. G. J. Am. Chem. Soc. 1982, 104, 848.

46. Johnstone, R. A. W.; Rose, M. E. I. Chem. Soc. Chem. Commun. 1983, 1269.

47. Zhang, H.; Chu, I. H; Leming, S.; Dearden, D. V. I. Am. Chem. Soc, 1991, 113, 7415.

48. Maleknia, S.; Brodbelt, J. S. J. Am. Chem. Soc., in press.

49. Greco, F.; Liguori, A.; Sindona, G.; Uccella, N. I. Am. Chem. Soc. 1990, 112, 9092.

50. Bojesen, G. J. Am. Chem. Soc. 1987, 109, 5557.

51. Massauz, J.; Desreux, J. F.; Duyckaerts, G. J. Chem. Soc. Dalton Trans. 1980, 865.

52. Takeda, Y.; Yano, H.; Ishibashi, M.; Isozumi, H. Bull. Chem. Soc. Ipn. 1980, 33, $/ 2$.

53. Lamb, J. D.; Izatt, R. M.; Swain, S, W.; Christensen, J. J. J. Am. Chem. Soc. 1980, 102, 475 . 